\title{
Dynamic Contrast-Enhanced Magnetic Resonance Imaging of Ocular Melanoma as a Tool to Predict Metastatic Potential
}

\author{
Wenbo Wei, PhD, * Guang Jia, PhD, *广 Hendrik von Tengg-Kobligk, MD, * $\neq$ \\ Johannes T. Heverhagen, MD, PhD, *\$ Mohamed Abdel-Rahman, MD, PhD, $\S$ Lai Wei, PhD, // \\ John B. Christoforidis, MD, $\$ \mathbb{T}$ Frederick Davidorf, MD, $\S$ and Michael V. Knopp, MD, PhD*
}

\begin{abstract}
Purpose: This study explores the capability of dynamic contrast-enhanced magnetic resonance imaging (DCE-MRI) to differentiate tumor characteristics of metastatic and nonmetastatic choroidal melanoma as a potential tool for patient management.

Materials and Methods: A total of 13 patients ( $69 \pm 9$ years) with choroidal melanoma were imaged using DCE-MRI on a 3-T MRI system with a 16-channel head coil. The Tofts 2-compartment model was chosen for quantification, and parameters $K^{\text {trans }}$ (the transfer constant from the blood plasma to the extracellular space) and $K_{\text {ep }}$ (the transfer constant from the extracellular space to the blood plasma) were calculated and compared. Metastasis was excluded by subsequent clinical work-up or confirmed by histology after targeted biopsy.

Results: Six patients were diagnosed with metastatic melanoma and 7 without. All orbital tumors were at least larger than $2 \mathrm{~mm}$. A significant difference was identified in $K^{\text {trans }}$ between patients with $(0.73 \pm 0.18 / \mathrm{min})$ and without $(1.00 \pm 0.21 / \mathrm{min})$ metastatic melanoma $(P=0.03)$, whereas the difference was not significantly shown in $K_{\text {ep }}(2.58 \pm 1.54 / \mathrm{min}$ of metastatic patients vs $2.98 \pm 1.83 / \mathrm{min}$ of nonmetastatic patients, $P=0.67$ ). Conclusions: Dynamic contrast-enhanced magnetic resonance imaging has the potential to differentiate orbital melanomas with metastatic and nonmetastatic spread. Thus, DCE-MRI has the potential to be an in vivo imaging technique to predict early which patients are prone to metastatic disease.
\end{abstract}

Key Words: choroidal melanoma, metastasis, dynamic contrast-enhanced magnetic resonance imaging, $K^{\text {trans }}$

(J Comput Assist Tomogr 2017;00: 00-00)

$\mathrm{M}$ alignant melanoma of the choroid is the most common type of primary intraocular malignancy in adults. It is caused by the uncontrolled pigmented cells growing in the eye. In the United States, the yearly mean incidence of choroidal melanoma is reported to be 6 to 7 cases per million people. Choroidal melanoma is more commonly seen in an older age group, with a progressively rising age-specific incidence rate that peaks at the age of 70 years. At the time of diagnosis, $99.5 \%$ of the patients have "no evidence of metastatic disease." 1 After treatment, either enucleation or

From the *Wright Center of Innovation in Biomedical Imaging and Department of Radiology, The Ohio State University, Columbus, OH; †Department of Physics and Astronomy, Louisiana State University, Baton Rouge, LA; \$Department of Diagnostic, Interventional and Pediatric Radiology, Inselspital, University of Bern, Bern, Switzerland; §Department of Ophthalmology and Visual Science, and $\|$ Center for Biostatistics, Department of Biomedical Informatics, The Ohio State University, Columbus, OH; and $\uparrow$ Department of Ophthalmology and Vision Science, The University of Arizona Medical Center, Tucson, AZ. Received for publication October 14, 2016; accepted December 14, 2016.

Correspondence to: Michael V. Knopp, MD, PhD, Wright Center of Innovation in Biomedical Imaging and Department of Radiology, The Ohio State University, Columbus, OH 43210 (e-mail: knopp.16@osu.edu).

This study was supported by the Wright Center of Innovation in Biomedical Imaging, its development fund, the OSU Medical Center Imaging signature program, and the Ohio Third Frontier Funding ODSA TECH 09-028 and TECH 11-044.

Copyright (C) 2017 Wolters Kluwer Health, Inc. All rights reserved. DOI: 10.1097/RCT.0000000000000598 brachytherapy, by 5 years, $30 \%$ of these patients will have died from metastatic melanoma. ${ }^{2,3}$ More than $90 \%$ of patients with metastasis show liver involvement. ${ }^{4,5}$ Therefore, early detection of metastasis may reduce its spread rate and help in clinical treatment planning and therapy assessment. ${ }^{6}$

It is assumed that metastasis is via hematogenous spread. Rapid progress has been made in the development of dynamic contrast-enhanced magnetic resonance imaging (DCE-MRI) in oncology by providing, in addition to detailed morphology, ${ }^{7}$ at the same time, spatially resolved functional information. ${ }^{8-10}$ Over the past decades, it has been established that changes in angiogenesis are a key factor in tumor growth and development. ${ }^{11}$ Folberg et $\mathrm{al}^{12}$ and Mehaffey et $\mathrm{al}^{13}$ have found direct correlation between the microvasculature of malignant melanoma and survival rates. It has been shown that time-resolved MRI is able to noninvasively visualize characteristic pharmacokinetic patterns of a contrast agent passing through the microvasculature of tumors, which can provide useful prognostic information for cancer patients. ${ }^{14-16}$ Based on a mathematical model, pharmacokinetic parameters can be calculated using a dedicated postprocessing environment, which can be used to improve noninvasive tumor characterization. ${ }^{17}$ Thus, DCE-MRI could provide a more specific in vivo analysis of the microcirculation of choroidal tumors than previous methods. ${ }^{18}$ This has been demonstrated successfully in different kinds of tumors (eg, breast cancer) with MRI and other imaging modalities. ${ }^{19-22}$

The potential of DCE-MRI-derived parameters has been reported to be able to assess the metastatic potential of melanoma. ${ }^{23}$ The studies on human melanoma xenografts revealed the correlation between the tumor tissue blood transfer rate $\left(K^{\text {trans }}\right)$ and the tumor aggressiveness and the extent of hypoxia in animal models. Although the tumors in general have increased angiogenesis, the more aggressive metastatic tumors are likely to produce more blood vasculature. ${ }^{24}$ However, the high cell density with lack of oxygen and nutrition supply leads to less functional blood vessels. The potential that DCE-MRI may provide additional information at the molecular level was investigated in the study. In this retrospective study, patients with metastatic and nonmetastatic melanoma have been imaged to determine the quantitative assessment of dynamic contrast enhancement and examine its potential correlation to metastasis of the choroidal melanoma. The parameters $K^{\text {trans }}$ (the transfer constant from the blood plasma to the extracellular space) and $K_{\text {ep }}$ (the transfer constant from the extracellular space to the blood plasma) from the Tofts 2 -compartment model were compared between the 2 groups. $^{22,25}$

\section{MATERIALS AND METHODS}

\section{Patients}

More than 50 patients were referred by ophthalmologists for 3-T MRI from May 2006 to March 2012 in patients with intraocular space occupying lesions, and imaging included DCE-MRI. Thirteen patients ( 7 men and 6 women; age, $69 \pm 9$ years) with 
no renal dysfunction and those with a tumor diameter greater than $2 \mathrm{~mm}$ were included in this evaluation. The tumor volume was calculated by measuring the tumor volume in each consecutive slice and adding them together. A total of 14 ocular lesions were diagnosed. All patients previously agreed to participate in the research evaluation. All examinations were performed in accordance with the approval of the institutional review board. Subsequent to the eye MRI, the patients received clinical staging that included ophthalmologic examinations and other imaging studies. When a metastasis was noted, it was histologically verified after targeted biopsy.

\section{Dynamic Contrast-Enhanced MRI}

Magnetic resonance imaging was performed using a 3-T whole-body MRI system (Achieva; Philips, Cleveland, Ohio) using a 32-channel phased-array head coil. Before performing DCE-MRI, $\mathrm{T}_{1}$-weighted images at coronal, sagittal, and axial planes, axial $\mathrm{T}_{2}$-weighted images, $3 \mathrm{D} \mathrm{T}_{1}$-weighted high resolution images, and axial diffusion-weighted images were acquired for reference. For the dynamic imaging, a coronal $3 \mathrm{D} \mathrm{T}_{1}$-weighted fast field echo sequence (also known as $\mathrm{T}_{1}$-weighted spoiled gradient echo) was acquired with fat suppression. Other parameters were $\mathrm{TR} / \mathrm{TE}=4.2 / 2.2$ milliseconds, flip angle $=15$ degrees, field of view $=180 \times 180 \times 32 \mathrm{~mm}^{3}$, voxel size $=0.9 \times 0.9 \times 2 \mathrm{~mm}^{3}$, temporal resolution $=9$ seconds, oversampling factor $=1.28$, turbo factor $=50$, number of slices $=16$, number of signal average $=1$, and no parallel imaging. After the start of the dynamic MR acquisition, 5 to 8 scans were made to serve as baseline before contrast injection. A total of 50 dynamic temporal points were acquired. The contrast agent Magnevist (Gd-DTPA ${ }^{2-}$; Bayer, Germany) was administered intravenously at a standard bolus dose of $0.1 \mathrm{mmol} / \mathrm{kg}$ body weight at a rate of $0.6 \mathrm{~mL} / \mathrm{s}$ immediately followed by a $15-\mathrm{mL}$ saline flush. ${ }^{9}$ The total of DCE-MRI acquisition was made within 6 to 8 minutes, depending on the slice number. The patients were instructed to keep their eyes closed and limit eyeball movement during the scan.
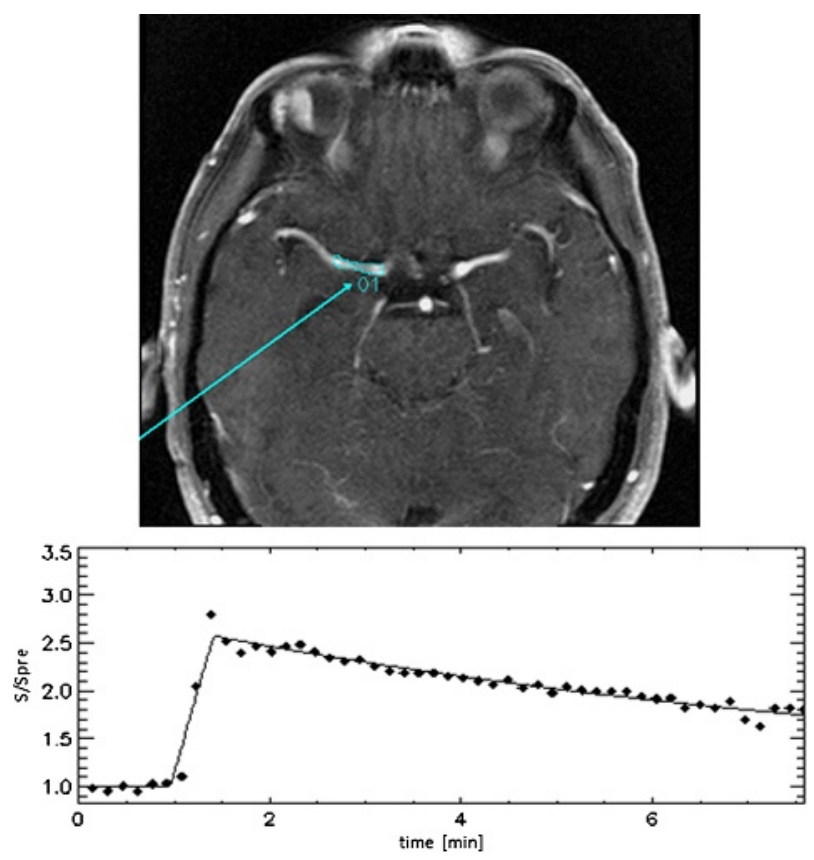

FIGURE 1. The AIF of ophthalmic artery at the same side of the choroidal lesion. Patient P07 was chosen, and the image at the 21 st temporal point was shown.
TABLE 1. Patient Demographic Information

\begin{tabular}{|c|c|c|c|c|}
\hline $\begin{array}{l}\text { Patient } \\
\text { No. }\end{array}$ & Sex & $\begin{array}{c}\text { Age at } \\
\text { Examination }\end{array}$ & $\begin{array}{l}\text { Ocular Tumor } \\
\text { Volume, } \mathbf{m m}^{3}\end{array}$ & $\begin{array}{l}\text { Metastasis } \\
\text { Information }\end{array}$ \\
\hline P01 & Female & 48 & 146 & No \\
\hline P02 & Male & 78 & 813 & No \\
\hline $\mathrm{P} 03$ & Male & 56 & 505 & No \\
\hline P04 & Female & 75 & 572 & No \\
\hline P05 & Female & 75 & 193 & No \\
\hline \multirow[t]{2}{*}{ P06 } & Female & 69 & 1453 & $\begin{array}{l}\text { Metastatic lesion } \\
\text { within the spine } \\
\text { and the liver }\end{array}$ \\
\hline & & & $2476^{*}$ & \\
\hline P07 & Male & 69 & 2116 & $\begin{array}{l}\text { Metastatic liver } \\
\text { lesion }\end{array}$ \\
\hline P08 & Male & 66 & 1462 & $\begin{array}{l}\text { Metastatic liver } \\
\text { lesion }\end{array}$ \\
\hline P09 & Male & 72 & 861 & No \\
\hline $\mathrm{P} 10$ & Female & 78 & 1624 & No \\
\hline P11 & Male & 70 & 4677 & $\begin{array}{l}\text { Metastatic liver } \\
\text { lesion }\end{array}$ \\
\hline $\mathrm{P} 12$ & Male & 59 & 274 & $\begin{array}{l}\text { Metastatic liver } \\
\text { lesion }\end{array}$ \\
\hline P13 & Female & 78 & 7763 & $\begin{array}{l}\text { Metastatic liver } \\
\text { lesion }\end{array}$ \\
\hline
\end{tabular}

The ocular tumor volume and the sites of metastasis are listed.

*Patient has a second ocular tumor.

\section{Tofts Model-Based Postprocessing}

The dynamic MR image data sets were postprocessed using in-house-developed software based on the Interactive Data Language (Exelis Visual Information Solutions, Boulder, Colo) environment. The pharmacokinetic analysis was applied to the contrast-enhanced signal based on the Tofts 2-compartment model. ${ }^{22}$ The region of interest (ROI) of the ophthalmic artery at the same side of the choroidal lesion was used to calculate the arterial input function (AIF) (Fig. 1). The subsequent output color map was calculated automatically. The DCE-MRI parameters $K^{\text {trans }}$ (the transfer constant from the blood plasma to the extracellular space) and $K_{\mathrm{ep}}$ (the transfer constant from the extracellular space to the blood plasma) were obtained. The contours of the primary site of the choroidal melanoma in the central slice were manually drawn for the ROI analysis. The ROIs were drawn by a clinical scientist with more than 5 years of experience in musculoskeletal research. The necrotic areas were excluded from the ROIs based on the axial $\mathrm{T}_{2}$-weighted images.

\section{Statistical Analysis}

The MRI quantitative parameters $K^{\text {trans }}$ and $K_{\text {ep }}$ of the primary sites were tested for normality using KolmogorovSmirnov test. Because $K^{\text {trans }}$ and $K_{\text {ep }}$ were normally distributed $(P>0.15)$, they were compared between the metastatic and nonmetastatic patients using 2 sample $t$ tests. A $P$ value of less than 0.05 was considered to represent a significant difference. Statistical analysis was performed using Statistical Analysis System v. 9.4 (SAS Institute, Cary, NC).

\section{RESULTS}

Of the total 13 patients with eye melanoma, 6 patients were diagnosed with metastatic lesions at various locations confirmed 

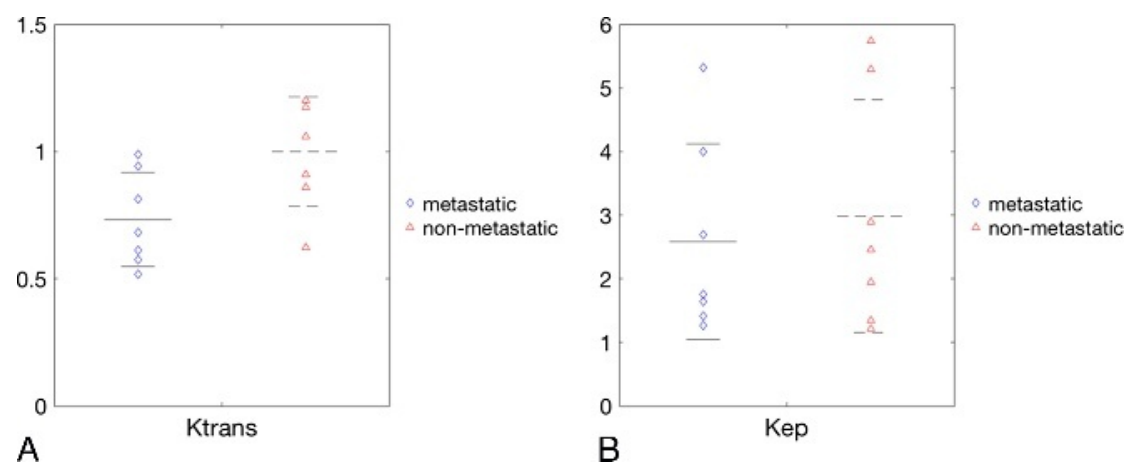

FIGURE 2. The distribution of $K^{\text {trans }}(\mathrm{A})$ and $K_{\mathrm{ep}}(\mathrm{B})$ according to the biopsy diagnosis on metastatic and nonmetastatic patients.

with histologic diagnosis. It revealed that 5 patients had metastatic liver lesions and 1 patient had metastatic lesions in both the spine and the liver, whereas the remaining 7 cases did not show any metastasis. The patients with and without metastasis were divided into 2 groups, and the DCE-MRI parameters were compared.

Table 1 lists the patient demographic information. The ocular tumor volume sizes were compared between the nonmetastatic and metastatic patients. Although no significant difference could be confirmed, the metastatic tumors trended to have a greater volume on average than nonmetastatic tumors $(2889 \pm 2538$ vs $\left.674 \pm 501 \mathrm{~mm}^{3}, P=0.06\right)$. The location of metastasis is listed for each patient.

The results of $K^{\text {trans }}$ and $K_{\mathrm{ep}}$ comparison between the metastatic and nonmetastatic patients are plotted in Figure 2. The mean and SD values of $K^{\text {trans }}$ and $K_{\text {ep }}$ are presented using solid and dashed lines. The $K^{\text {trans }}$ of metastatic patients $(0.73 \pm 0.18 / \mathrm{min}$, $\mathrm{n}=6)$ are statistically lower than the nonmetastatic ones $(1.00 \pm 0.21 / \mathrm{min}, \mathrm{n}=7)(P=0.03)$, which means that the metastatic patient's eye lesion has a higher contrast agent transfer from blood plasma to extracellular space. No significant difference was found in $K_{\text {ep }}(2.58 \pm 1.54 / \mathrm{min}$ of metastatic patients vs $2.98 \pm 1.83 / \mathrm{min}$ of nonmetastatic patients) $(P=0.67)$.
The representative $T_{1}$-weighted MRI images of patients with metastatic and nonmetastatic lesions in the DCE-MRI series are shown in Figure 3 upper row, the associated time-intensity curves in the bottom row. On the longitudinal axis, the signal intensity at each time point was normalized to the average signal intensity before the contrast agent wash in. For the postcontrast $T_{1}$ images, the time point was chosen when the contrast agent washed in and reached the peak value. The eye tumor without metastasis shows a much higher contrast agent uptake value that makes it brighter on the $T_{1}$ image. The ophthalmic arteries presented with intense enhancement and were used for the calculation of the AIF. In the time-intensity curves, the nonmetastatic tumor has a rapidly increasing slope that corresponds to the higher $K^{\text {trans }}$ values than found in nonmetastatic patients. The peak value was found to be approximately 2.11 for the nonmetastatic and 1.32 for the metastatic tumor.

The $K^{\text {trans }}$-coded color maps of 1 patient with metastasis and 1 patient without metastasis are presented in Figure 4. In the metastatic patient, a large spinal metastasis was found within the L4 vertebral body, and an intermediate subcentimeter lesion was found within the L3 vertebral body. The ocular lesion with metastasis presents heterogeneous $K^{\text {trans }}$ distribution with an
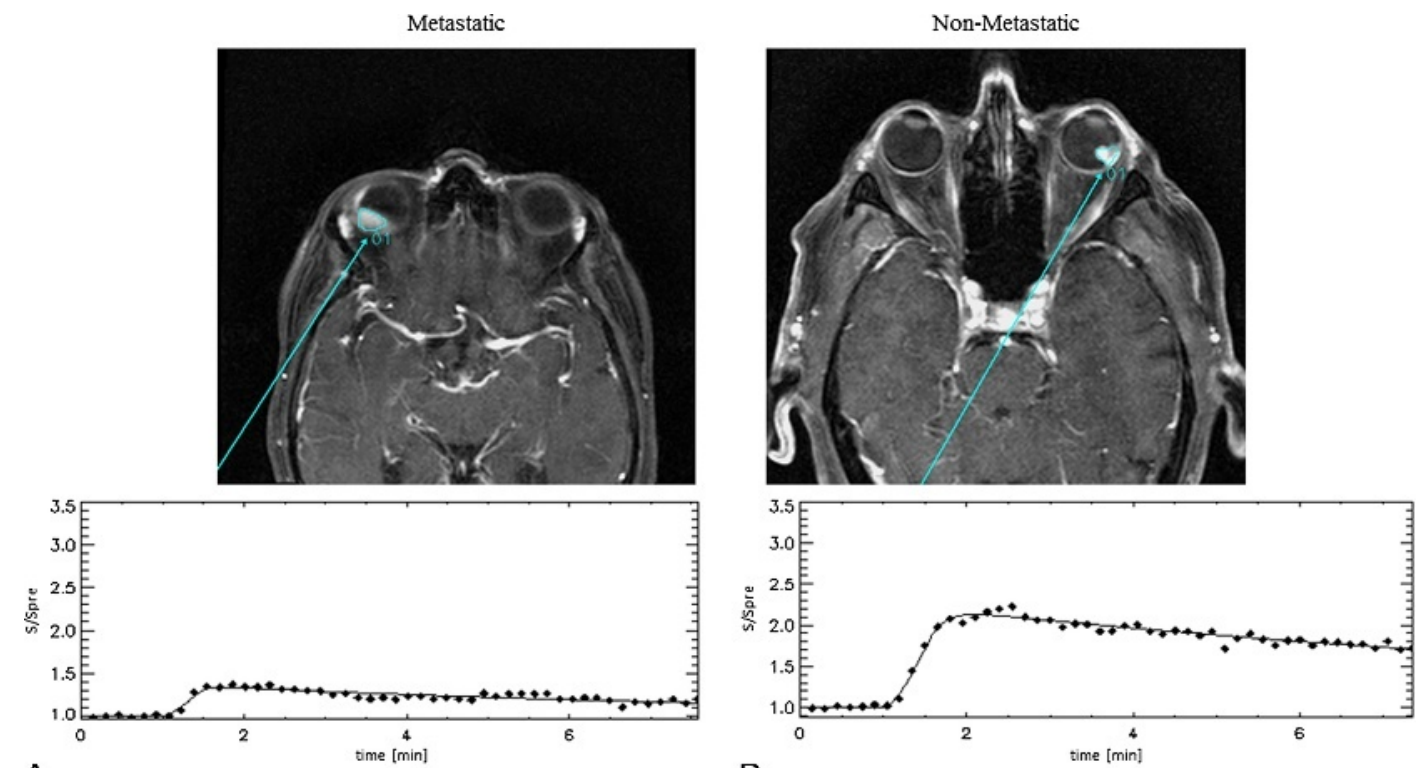

A

B

FIGURE 3. The time-intensity curves for metastatic (A, patient P06, 10th temporal point) and nonmetastatic (B, patient P09, 12th temporal point) patients. Region of interests were manually drawn on the DCE-MRI images. 


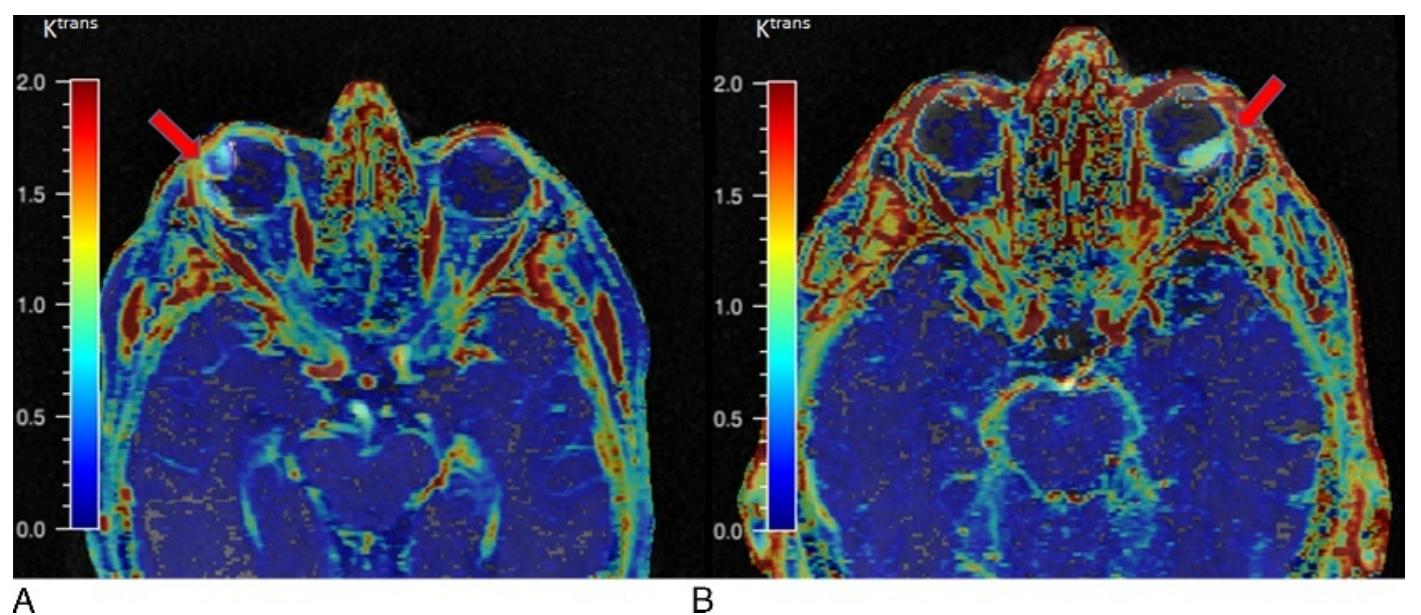

FIGURE 4. The $K^{\text {trans }}$-coded color maps of metastatic (A, patient P06) and nonmetastatic (B, patient P05) patients were shown.

average value of approximately $0.61 / \mathrm{min}$. The lesion with no metastasis shows higher $K^{\text {trans }}$ values with an average of $1.17 / \mathrm{min}$. High $K^{\text {trans }}$ values are also seen in the blood vessels.

\section{DISCUSSION}

Current cross-sectional imaging techniques have evolved with improved spatial resolution to enable noninvasive morphologic imaging of ocular and orbital structures. Nevertheless, the ability to distinguish between tumors with metastasis and without metastasis remains a challenge. To overcome this, additional indepth tumor microcirculatory information needs to be obtained. Malignant melanoma is the tumor entity with the fastest rise in incidence, and also, noninvasive imaging approaches as needed because of the inability to readily biopsy the eye. Malignant melanoma is known to have a high expression of vascular endothelial growth factor. Dynamic contrast-enhanced magnetic resonance imaging is known to reveal different contrast enhancement patterns in relation to microvasculature and expression of angiogenetic factor such as vascular endothelial growth factor. $^{23,26,27}$ The study is innovative because so far, very few studies have applied pharmacokinetic modeling such as DCEMRI to the ocular and orbital region in vivo.

This study shows that $K^{\text {trans }}$ is significantly lower in the metastatic than the nonmetastatic melanoma. When the contrast agent is injected into the blood vessels, it is more difficult for the contrast agent to diffuse into the extracellular space in the ocular melanoma that leads to metastatic spread. It may indicate that the blood vessels in the metastatic melanoma are less functional than the nonmetastatic melanoma. Although more blood vessels are needed for cancer cells to spread to other areas of the body and form new tumors at metastatic sites, they seem to have less patency and limited blood perfusion for oxygen and substrates.

It has been reported by Chikui et $\mathrm{al}^{28}$ that the $K^{\text {trans }}$ decrease significantly correlated with the progression of the $\mathrm{N}$ stage in clinical patients with oral squamous cell carcinoma. They speculated that the reduced $K^{\text {trans }}$ and blood flow may lead to the hypoxic state and higher likelihood of metastasis. $\mathrm{Li}$ et $\mathrm{al}^{29}$ conducted DCE-MRI studies on 2 types of melanoma xenografts, including the most metastatic, C8161, and the least metastatic, A375P. Statistically significant higher $K^{\text {trans }}$ was observed in A375P compared with $\mathrm{C} 8161$, which showed that $K^{\text {trans }}$ in the tumor could help distinguish between highly metastatic and least metastatic melanomas. Similar results were found in the research by an independent group. ${ }^{30} \mathrm{CK}-160$ cervical carcinoma and V-27 melanoma xenografts were used as tumor models, and parametric images of $K^{\text {trans }}$ and $v_{\mathrm{e}}$ were produced. It showed that highly metastatic tumors presented lower $K^{\text {trans }}$ values $(P=0.00033)$, whereas $\nu_{\mathrm{e}}$ showed lower values, but not significant, between metastatic and nonmetastatic tumors $(P>0.05)$. Those observations are consistent with our results obtained in ocular melanoma patients.

One limitation of this study is the relatively small number of subjects. One of the reasons is because the patients with lesion diameter less than $2 \mathrm{~mm}$ were excluded to avoid significant partial volume effect in the analysis. It limited the statistical power of the results and made the SD of data analysis large. The second limitation is that only the central slice through the tumor was chosen for analysis. Although the tumor center was expected to be included, the ROI was not able to display the $K^{\text {trans }}$ distribution throughout the whole tumor. An ROI covering the entire tumor except the necrotic area may be used to generate $K^{\text {trans }}$. Because only a single observer defined the ROI contours, the interobserver reliability was not tested. Another limitation is tumor-related other factors were not considered, such as the degree of primary tumor. Those characteristics may contribute to the $K^{\text {trans }}$ findings. Recent publications have shown that variations were found in $K^{\text {trans }}$ and $v_{\mathrm{e}}$ calculations using different analysis software packages ${ }^{31}$; we used our well-established and validated in-house-developed Interactive Data Language environment. ${ }^{9}$ More analysis needs to be done using different software to explore the intersoftware consistency.

\section{CONCLUSIONS}

It was shown that DCE-MRI can differentiate the tumor characterization between metastatic and nonmetastatic melanoma by the $K^{\text {trans }}$ value. Thus, DCE-MRI has the potential to be an in vivo imaging technique to indicate which patients are prone to metastatic disease and may be an appropriate tool to stratify therapeutic approaches.

\section{REFERENCES}

1. AnonymousMortality in patients with small choroidal melanoma. COMS report no. 4. The Collaborative Ocular Melanoma Study Group. Arch Ophthalmol. 1997;115:886-893.

2. AnonymousThe Collaborative Ocular Melanoma Study (COMS) randomized trial of pre-enucleation radiation of large choroidal melanoma II: initial mortality findings. COMS report no. 10. Am J Ophthalmol. 1998; 125:779-796. 
3. Diener-West M, Earle JD, Fine SL, et al. The COMS randomized trial of iodine 125 brachytherapy for choroidal melanoma, III: initial mortality findings. COMS Report No. 18. Arch Ophthalmol. 2001;119:969-982.

4. Singh AD, Bergman L, Seregard S. Uveal melanoma: epidemiologic aspects. Ophthalmol Clin North Am. 2005;18:75-84. viii.

5. Lorigan JG, Wallace S, Mavligit GM. The prevalence and location of metastases from ocular melanoma: imaging study in 110 patients. $A J R \mathrm{Am}$ J Roentgenol. 1991;157:1279-1281.

6. Hui KH, Pfeiffer ML, Esmaeli B. Value of positron emission tomography/computed tomography in diagnosis and staging of primary ocular and orbital tumors. Saudi J Ophthalmol. 2012;26:365-371.

7. Chambers RB, Davidorf FH, McAdoo JF, et al. Magnetic resonance imaging of uveal melanomas. Arch Ophthalmol. 1987;105:917-921.

8. Hoffmann U, Brix G, Knopp MV, et al. Pharmacokinetic mapping of the breast: a new method for dynamic MR mammography. Magn Reson Med. 1995;33:506-514.

9. Knopp MV, Giesel FL, Marcos H, et al. Dynamic contrast-enhanced magnetic resonance imaging in oncology. Top Magn Reson Imaging. 2001; 12:301-308

10. Rodjan F, de Graaf P, van der Valk P, et al. Retinoblastoma: value of dynamic contrast-enhanced MR imaging and correlation with tumor angiogenesis. AJNR Am J Neuroradiol. 2012;33:2129-2135.

11. Folkman J. The role of angiogenesis in tumor growth. Semin Cancer Biol. 1992;3:65-71.

12. Folberg R, Chen X, Boldt HC, et al. Microcirculation patterns other than loops and networks in choroidal and ciliary body melanomas. Ophthalmology. 2001;108:996-1001.

13. Mehaffey MG, Gardner LM, Folberg R. Distribution of prognostically important vascular patterns across multiple levels in ciliary body and choroidal melanomas. Am J Ophthalmol. 1998;126:373-378.

14. Knopp MV, Weiss E, Sinn HP, et al. Pathophysiologic basis of contrast enhancement in breast tumors. J Magn Reson Imaging. 1999;10:260-266.

15. Mayr NA, Hawighorst H, Yuh WT, et al. MR microcirculation assessment in cervical cancer: correlations with histomorphological tumor markers and clinical outcome. J Magn Reson Imaging. 1999;10:267-276.

16. Padhani AR, Husband JE. Dynamic contrast-enhanced MRI studies in oncology with an emphasis on quantification, validation and human studies. Clin Radiol. 2001;56:607-620.

17. Port RE, Knopp MV, Hoffmann U, et al. Multicompartment analysis of gadolinium chelate kinetics: blood-tissue exchange in mammary tumors as monitored by dynamic MR imaging. J Magn Reson Imaging. 1999;10: 233-241.

18. Nagashima T, Suzuki M, Yagata $H$, et al. Dynamic-enhanced MRI predicts metastatic potential of invasive ductal breast cancer. Breast Cancer. 2002;9: 226-230.
19. Choyke PL, Knopp MV, Libutti SK. Special techniques for imaging blood flow to tumors. Cancer J. 2002;8:109-118.

20. Knopp MV, Brix G, Junkermann HJ, et al. MR mammography with pharmacokinetic mapping for monitoring of breast cancer treatment during neoadjuvant therapy. Magn Reson Imaging Clin N Am. 1994;2:633-658.

21. Yuan Y, Kuai XP, Chen XS, et al. Assessment of dynamic contrast-enhanced magnetic resonance imaging in the differentiation of malignant from benign orbital masses. Eur J Radiol. 2013;82:1506-1511.

22. Tofts PS. Modeling tracer kinetics in dynamic Gd-DTPA MR imaging. J Magn Reson Imaging. 1997;7:91-101.

23. Ovrebo KM, Ellingsen C, Galappathi K, et al. Dynamic contrast-enhanced magnetic resonance imaging of the metastatic potential of melanoma xenografts. Int J Radiat Oncol Biol Phys. 2012;83:e121-e127.

24. Leenders W, Kusters B, Pikkemaat J, et al. Vascular endothelial growth factor-A determines detectability of experimental melanoma brain metastasis in GD-DTPA-enhanced MRI. Int J Cancer. 2003;105: $437-443$.

25. Tofts PS, Brix G, Buckley DL, et al. Estimating kinetic parameters from dynamic contrast-enhanced T(1)-weighted MRI of a diffusable tracer: standardized quantities and symbols. J Magn Reson Imaging. 1999;10: 223-232.

26. Kim JH, Im GH, Yoon J, et al. Dynamic contrast-enhanced MRI for assessing therapeutic response of choroidal neovascularization in a rat model. Invest Ophthalmol Vis Sci. 2012;53:7693-7700.

27. Lee L, Sharma S, Morgan B, et al. Biomarkers for assessment of pharmacologic activity for a vascular endothelial growth factor (VEGF) receptor inhibitor, PTK787/ZK 222584 (PTK/ZK): translation of biological activity in a mouse melanoma metastasis model to phase I studies in patients with advanced colorectal cancer with liver metastases. Cancer Chemother Pharmacol. 2006;57:761-771.

28. Chikui T, Kitamoto E, Kami Y, et al. Dynamic contrast-enhanced MRI of oral squamous cell carcinoma: a preliminary study of the correlations between quantitative parameters and the clinical stage. Br J Radiol. 2015; 88:20140814.

29. Li LZ, Zhou R, Xu HN, et al. Quantitative magnetic resonance and optical imaging biomarkers of melanoma metastatic potential. Proc Natl Acad Sci U S A. 2009;106:6608-6613.

30. Ovrebo KM, Ellingsen C, Hompland T, et al. Dynamic contrast-enhanced magnetic resonance imaging of the metastatic potential of tumors: a preclinical study of cervical carcinoma and melanoma xenografts. Acta Oncol. 2013;52:604-611.

31. Beuzit L, Eliat PA, Brun V, et al. Dynamic contrast-enhanced MRI: study of inter-software accuracy and reproducibility using simulated and clinical data. J Magn Reson Imaging. 2016;43:1288-1300. 